Nig. J. Biotech. Vol. 37(1): 138-149 (June 2020)

ISSN: 01891731

Available online at

http://www.ajol.info/index.php/njb/index

and www.biotechsocietynigeria.org

DOI: https://dx.doi.org/10.4314/njb.v37i1.15

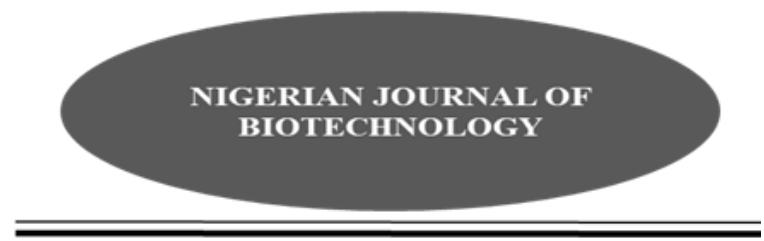

\title{
FAMEs Profile of Oil Produced by Oleaginous Fungi Isolated from Fermented Beverage Wastewaters and Soil
}

\author{
Nsa, I.Y., Adeloye, G.B., Odunsi, A. A., Akinyemi, B.T. ,Tubonemi, J.T., Saliu, \\ M. O. and Adepoju, J.P
}

Department of Microbiology, Faculty of Science, University of Lagos

\begin{abstract}
Fungal strains isolated from fermented maize (ogi) (PW) and sorghum-based brewery wastewaters (BW) and two soil isolates were evaluated for oleaginicity. The fungal isolates from the wastewater that had lipid content of at least $20 \%$ of their biomass were identified by both culture methods and internal transcribed spacer (ITS) 1-5.8S-ITS2 ribosomal DNA sequencing. The isolates were identified to be Aspergillus fumigatus (PW8), Aspergillus flavus (PW10), Candida tropicalis (PW16) and Aspergillus tubingensis (PW3), Trichosporon luoberi (BW7), Aspergillussp. (BW4) and Candida tropicalis(BW1; BW3). FAMEs composition was determined for the four strains with the highest lipid content by acidcatalyzed transesterification and analyzed by Gas Chromatography-Flame Ionization Detector (GC-FID). Palmitoleic acid was the dominant fatty acid in M. circinelloides and T. reesei, and the best producers of capric and lauric acids were Aspergillus fumigatus and Aspergillus sp. (BW4), respectively. These fatty acids are beneficial in making cosmetics and pharmaceuticals (antimicrobials and dietary supplements). The analysis of the FAMEs profile in the species indicated low amounts or absence of some key long chain fatty acid (LCFA) constituents of biodiesels. Based on the FAMEs profile of $M$. circinelloides investigated, this strain could hold promise for use as feedstock for biodiesel with genetic engineering and a tailored lipid production favouring enrichment of LCFA.
\end{abstract}

Keywords: Fungal lipids, wastewater, fatty acid methyl ester, GC-FID

Corresponding author: insa@unilag.edu.ng

\section{Introduction}

Filamentous fungi have broad application as production hosts in the industry mainly for their capacity to secrete metabolites (Peberdy, 1994, Punt et al., 2002; Wosten, 2019). Microbial oils are secondary metabolites produced by bacteria, yeast, fungi and microalgae and are accumulated within specific organelles like lipid bodies within the cell (Kosa and Ragauskas, 2011). A microorganism is said to be oleaginous when it can accumulate more than $20 \%$ of biomass as lipids (Ratledge and Wynn,
2002; Thevenieau and Nicaud, 2013). Microbial systems that can produce and store oil have attracted significant research attention in recent years (Bharathiraja et al., 2017), especially triacylglycerols (TAGs) produced from oleaginous microorganisms as the supplementary sources of conventional oil for biodiesel production (Thevenieau and Nicaud, 2013). Oleaginous fungi include Mucor circinelloides, Candida tropicalis, Pythium ultimum, Morietella isabellina, Aspergillus terreus, Claviceps purpurea, Pellicularia praticola (Thevenieau and 
Nsa et al./ Nig. J. Biotech. Vol. 37 Num. 1 : 138-149 (June 2020)

Nicaud, 2013). These fungi can also grow by utilizing sugarcane molasses, soluble starch and wheat straw (Lin et al., 2010). Nitrogen limitations in the substrate are required for the accumulation of lipids (Ratledge 2002; Calvey et al., 2016).

Microbial oil extraction processes are simpler to handle and require less labor ( $\mathrm{Li}$ and Wang, 1997; Li et al., 2008) compared to plant oil extraction procedures. Plant oil extraction requires the processing of the specific plant tissue (Atabani et al., 2013) as opposed to microbial oil extraction in the aqueous phase or ultrasonication (Zhang et al., 2014). The procedure for oil extraction is too expensive to apply for production on a large scale, therefore the viability of production of microbial oil is dependent on the high conversion of substrate to intracellular lipids combined with high extraction efficiency at low energy consumption (Bharathiraja et al., 2017).

Biodiesels are defined as the fatty acid alkyl monoesters (FAMEs) derived from renewable sources, such as vegetable oils and animal fats. They are produced by transesterification of the lipid with an alcohol especially methanol (Yap et al., 2011). The environmental benefits of using biodiesel over petroleum diesel include biodegradability, lower sulfur and aromatic hydrocarbon content and the reduction in the emissions of carbon monoxide, carbon dioxide and particulate matter (Meher et al., 2006; Sawangkeaw and Ngamprasertsith, 2013). The main disadvantage of this process is the unavailability of feedstocks and substrates, which accounts for $70 \%$ of the final cost of the biodiesel (Hanna, 1991; Thevenieau and Nicaud, 2013).

There is an increase in the demand for renewable fuel due to the limited energy resources and the environmental hazards that fossil fuels pose to the environment. Biodiesel is being increasingly investigated as an alternative to fossil fuels in modified combustion engines for transport as well as in engines for power generation (Gavrilescu and Chisti, 2005; Bharathiraja et al., 2014).

The composition and the yield of microbial oils are affected by cultural conditions such as substrates, incubation period, nitrogen source, $\mathrm{pH}$ and aeration of the culture medium (Liu et al., 2010). The yield and type of lipid are dependent on several factors like the type of organism, culture conditions and the substrate chosen (Ledesma-Amaro et al., 2016). The use of pure fungal isolates from environmental sources as feedstock for the production of fatty acid methyl esters relevant in biodiesel, and pharmaceuticals and cosmetics was investigated.

\section{Materials and Methods}

Source of fungal isolates

Mucor circinelloides (IYN 13) and Trichoderma reesei (IYN 15) are laboratory stock strains (unpublished data) that were isolated from the soil. These strains were selected because of other reports on their oleaginicity. All the other fungi from this study were obtained during the screening of untreated wastewater from two sources- fermented cereal "ogr" (PW) and a brewery (BW) in Ogun State, Nigeria. The brewery uses sorghum as the substrate for its fermentation process and corn was the substrate for ogi. The wastewater samples were collected in sterile 1 litre plastic bottles and were processed within 24 hours of sample collection.

Enrichment and isolation of pure isolates from the wastewater samples

Ten (10) $\mathrm{ml}$ of the wastewater sample was added into a $250 \mathrm{ml}$ flask containing $50 \mathrm{ml}$ sterilized enrichment medium. Enrichment medium was composed of $(\mathrm{g} / \mathrm{l}) \mathrm{D}$ - xylose $100 \mathrm{~g}$, yeast extract $1 \mathrm{~g}$, $\mathrm{KH}_{2} \mathrm{PO}_{4} 2.0 \mathrm{~g}, \mathrm{MgSO}_{4} 7 \mathrm{H}_{2} \mathrm{O} 0.75 \mathrm{~g}, \mathrm{Na}_{2} \mathrm{HPO}_{4} 1 \mathrm{~g}$, $\mathrm{CaCl}_{2} .2 \mathrm{H}_{2} \mathrm{O} 0.2 \mathrm{~g}, \mathrm{FeCl}_{3} 0.01 \mathrm{~g}, \mathrm{ZnCl}_{2} 0.1 \mathrm{~g}$, Rose Bengal $50 \mathrm{mg} / \mathrm{L}, 3.3 \mathrm{ml}$ of chloramphenicol solution $(10000 \mathrm{U} / \mathrm{mL}$ ) and set at pH 7.0 (Abu-Elreesh and Abd-El-Haleem, 2014). The mixture of the wastewater and the enrichment medium was then cultured at $28^{\circ} \mathrm{C}$, with agitation using orbital shaker at $180 \mathrm{rpm}$ for 48 hours. Serial dilution was carried out on an aliquot of the incubated medium, and cultured on PDA (Himedia, India) supplemented with chloramphenicol and Rose Bengal Chloramphenicol agar (Oxoid, UK). Incubation was done at $35^{\circ} \mathrm{C}$ for 5 days. Pure cultures were then obtained from mixed culture plates. Morphological appearances of the inoculated plates (at room temperature) were observed and distinct colonies were subcultured to obtain pure isolates which were then maintained on Potato Dextrose Agar slants and stored at $-20^{\circ} \mathrm{C}$.

Culturing of the laboratory stock isolates

Isolates M. circinelloides (IYN 13) and T. reesei (IYN 15) were cultured on PDA +Cam (PDAC) and Rose Bengal Chloramphenicol (RBC) (Oxoid, UK).

Classical and Molecular identification of the fungal isolates

Cultural identification of the fungal isolates 
Nsa et al./ Nig. J. Biotech. Vol. 37 Num. 1 : 138-149 (June 2020)

The isolates were identified based on their growth patterns on PDAC and RBC and by microscopy. Pure cultures were obtained for all the strains and were stored on PDA slants

\section{Molecular identification of the fungal isolates}

The laboratory strains (IYN 13 and IYN 15) had been previously authenticated by morphological and molecular methods (unpublished). DNA extracted from all the other fungal strains was done using $Z R$ Fungal/Bacterial DNA MiniPrep ${ }^{\mathrm{TM}}$ kit (Zymo, USA), according to the manufacturer's instructions. The DNA extractions and sequencing analyse $s$ were performed at the University of Lagos. Polymerase Chain Reaction (PCR) of the extracted genomic DNAs from the 7 isolates was done in a GeneAmp PCR system 9700 PCR thermal cycler. Each $25 \mu$ master mix consisted of $2.5 \mu$ of $10 x$ PCR buffer, $1 \mu$ of $25 \mathrm{mM} \mathrm{MgCl} 2,1 \mu \mathrm{l}$ each of forward (ITS5F: GGAAGTAAAAGTCGTAACAAGG) and reverse (ITS4R: TCCTCCGCTTATTGATATG) primers (Inqaba, South Africa), $1 \mu \mathrm{l}$ of DMSO, $2 \mu \mathrm{l}$ of $2.5 \mathrm{mMdNTPs}, 0.1 \mu \mathrm{l}$ of $5 \mu \mathrm{g} / \mu \mathrm{l}$ Taq DNA polymerase, $3 \mu \mathrm{l}$ of $10 \mathrm{ng} / \mu \mathrm{l}$ DNA and $13.4 \mu \mathrm{l}$ Nuclease free water. The PCR conditions were as follows: Initial denaturation at $94^{\circ} \mathrm{C}$ for 5 mins, followed by 36 cycles of denaturation at $94^{\circ} \mathrm{C}$ for $30 \mathrm{~s}$, annealing at $54^{\circ} \mathrm{C}$ for $30 \mathrm{~s}$, elongation at $72^{\circ} \mathrm{C}$ for $45 \mathrm{~s}$, a final elongation step at $72^{\circ} \mathrm{C}$ for 7 mins and hold temperature at $10{ }^{\circ} \mathrm{C}$. The amplicons were visualized on Safe view-stained $1.5 \%$ agarose electrophoresis gels. The expected size of the amplicons was about $650 \mathrm{bp}$ and the DNA ladder used was Hyperladder ${ }^{\mathrm{TM}}$ $1 \mathrm{~kb}$ (Bioline, TN, USA).

The PCR amplicons were sequenced at the DNA Sequencing Facility of the Bioscience Center, International Institute of Tropical Agriculture, Ibadan, Oyo using 3130XL genetic analyzer (Applied Biosystems, CA, USA). The sequences were checked for quality and assembled using BioEdit (version 7.2.5) Sequence Alignment Editor (Hall, 1999). The consensus sequence obtained for each isolate was compared to the GenBank nucleotide data library using the Basic Local Alignment Search Tool, BLAST software (Altschul et al., 1990) at the National Centre for Biotechnology Information (NCBI (http://www.ncbi.nlm.nih.gov) website (Nsa et al., 2020). The sequences were submitted to GenBank and accession numbers have been assigned to the isolates.

\section{Microscopic screening for Lipid production}

The microscopic screening of the pure isolates for intracellular lipid accumulation was performed by a modified Sudan Black B method (Thancharoen et al., 2017). Yeast Extract Malt Extract Agar (YEMEA) of basal medium composition ( $\mathrm{g} / \mathrm{l})$ : glucose, 10; peptone, 5; yeast extract, 5; and malt extract, 3 and agar-agar 15 was used for culturing the organisms in glass tubes for 48 hours (Liu et al., 2010). The two-day-old isolates were smeared, heat-fixed, flooded with Sudan Black B stain and kept for 15 minutes until the stain turned yellowish-green. The stain was rinsed and counterstained with safranin for 30 seconds. It was thereafter air-dried, blotted and observed under a light microscope (Bresser LCD 40 x1400 Germany) at a100 x magnification. Intracellular lipid accumulation in fungal cells was determined based on the density of globules /retention of Sudan Black B in cells.

\section{Quantitative examination of lipid production in the isolates/Liquid production medium}

Lipid production medium was prepared as described by Abu-Elreesh and Abd-El-Haleem, (2014) containing (in $\mathrm{g} / \mathrm{L}$ ): yeast extract 0.5 , $\mathrm{MgSO}_{4} .7 \mathrm{H}_{2} \mathrm{O} 0.4, \mathrm{KH}_{2} \mathrm{PO}_{4} 2.0, \mathrm{CaCl}_{2} 0.5, \mathrm{CuSO}_{4} .5 \mathrm{H}_{2} \mathrm{O}$ 0.05 and sodium molybdate 0.005 ; and $5 \%$ glucose $(\mathrm{w} / \mathrm{v}), \mathrm{pH} 6$ and dispensed into $250 \mathrm{ml}$ Erlenmeyer flasks. Each flask was inoculated with a fungus (positive for intracellular lipid accumulation) and incubated at $28^{\circ} \pm 2^{\circ} \mathrm{C}$ with a shaking speed of 180 rpm for 5 days.

Effects of carbon sources on accumulation and FAMES composition of the isolates

Lipid production medium was prepared as described above and sucrose was used as an alternative carbon source to glucose. Thirty (30) $\mathrm{ml}$ of the medium was dispensed into $100 \mathrm{ml}$ Erlenmeyer flask in triplicates and inoculated with 5day old fungal cultures and incubated with shaking at $180 \mathrm{rpm}, 28^{\circ} \mathrm{C} \pm 2^{\circ} \mathrm{C}$ for 5 days. The fungal biomass was harvested from each culture .

Lipid Yield (dry weight of lipid, lipid extraction process and lipid content) Estimation Dry weight of the biomass: Samples were filtered using a preweighed sterile Whatman filter paper inserted in a funnel placed in conical flasks and allowed to drain completely. The biomass was washed twice with distilled water and then dried in a hot air oven at $70^{\circ} \mathrm{C}$ to a constant weight. The dry weights of the biomass were recorded and used for lipid extraction.

The lipid extraction process was carried out according to a modified Bligh and Dyer method (Muniraj et al., 2017). The dried fungal masses 
Nsa et al./ Nig. J. Biotech. Vol. 37 Num. 1 : 138-149 (June 2020)

were crushed in a mortar and pestle and centrifuged at 10,000 rpm for 20 mins. A mixture of chloroform, methanol and distilled water (2:1:1) was added to the centrifuged biomass and spun again for 20 mins. The lower liquid phase (chloroform layer containing lipid) was extracted and dispensed into pre-weighed Bijoux bottles and evaporated using nitrogen gas. The remaining lipids were weighed and recorded.

The lipid content and the biomass yield of each fungal isolate were estimated using the equations described by Muniraj et al., 2017.

The lipid content in biomass $=Y_{L / X}$,

$$
Y_{L / X}=\frac{L L}{x x}
$$

Where, $L$, maximum lipid yield, $g / L$ and $X$, biomass yield that corresponds to the volume of the medium.

Determination of Fatty-Acid Methyl Esters (FAMEs) profile by Gas Chromatography-Flame Ionization Detector (GC-FID)

The lipid extract from the fungal isolates was transesterified by the addition of methanol, concentrated hydrochloric acid and water in the ratio 10:1:1 as described by Patel et al., (2016); the mixture in glass tubes was centrifuged and the upper phase removed using hexane. The hexane phase was passed through a gas chromatographic column. Gas chromatography was carried out on the transesterified extract. To determine FAMEs, 5-point serial dilution calibration standards $(0.25,0.50,1.00$, $2.00,4.00 \mathrm{ppm}$ ) were prepared from the stock and used to calibrate the GC-FID. Determination of the levels of FAMEs was done using Agilent 7820A gas chromatography coupled to a flame ionization detector fitted with a DB-1 capillary column coated with 5\% Phenyl Methyl Siloxane (30m length $x$ $0.32 \mathrm{~mm}$ diameter $\times 0.25 \mu \mathrm{m}$ film thickness) (Agilent Technologies). After calibration, the samples were analyzed and chromatogram and its fatty acid concentrations obtained.

\section{Results}

Isolation and enumeration of pure fungal isolates from the wastewater samples.

A total of twenty-five fungal isolates were obtained from $10^{-5}$ PDA dilution plates of the brewery wastewater and fermented cereal ogi wastewater. The isolates from the brewery and ogi wastewaters were labelled BW and PW, respectively. From the mixed culture plates (Fig.1), nine isolates were obtained from the brewery wastewater and sixteen isolates from the ogi wastewater. The laboratory strains (previously isolated from the soil) $M$. circinelloides and $T$. reesei were maintained on PDA.
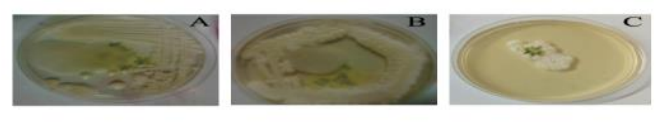

Figure 1. Some mixed fungal culture plates from A. Brewery Wastewater (dominated by yeasts) B. Ogi Wastewater (dominated by yeasts) and C. Ogi Wastewater (moulds).
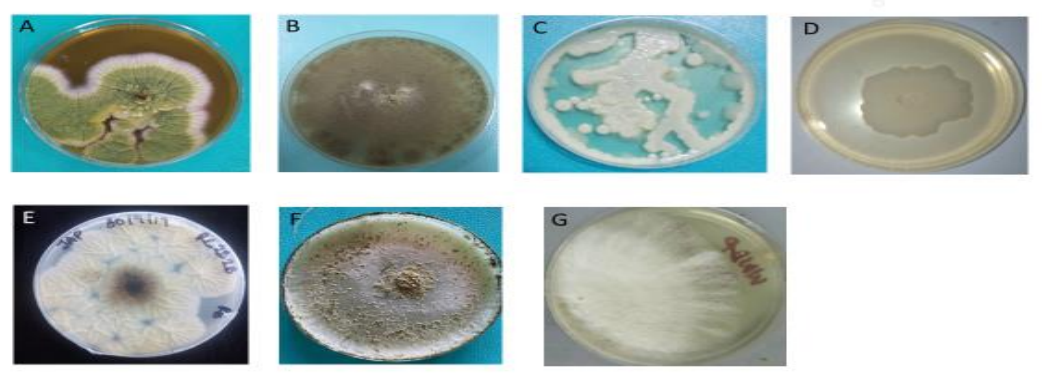

Figure 2. Pure cultures of fungal strains. A. Aspergillus flavus, B. Aspergillus

fumigatus, C. Candida tropicalis D. Candida tropicalis, E. Trichoderma reesei, F.

Mucor circinelloides, G. - Aspergillus sp. 
Nsa et al./ Nig. J. Biotech. Vol. 37 Num. 1 : 138-149 (June 2020)

Figure 2. Pure cultures of fungal species . A. Aspergillus flavus, B. Aspergillus fumigatus, C. Candida tropicalis D. Candida tropicalis, E. Trichoderma reesei, F. Mucor circinelloides, G. Aspergillus sp.

Microscopic Screening of isolates for lipid and Trichoderma reesei. The isolates BW1, BW3, accumulation.

Ten out of the 27 pure culture fungal isolates (Fig. 2) that were screened on YEMEA displayed significant oil accumulation including M. circinelloides BW4 and BW7, PW3, PW8, PW10 and PW16 were positive for oil accumulation based on the amount of Sudan black dye retained in their cells (Fig. 3)
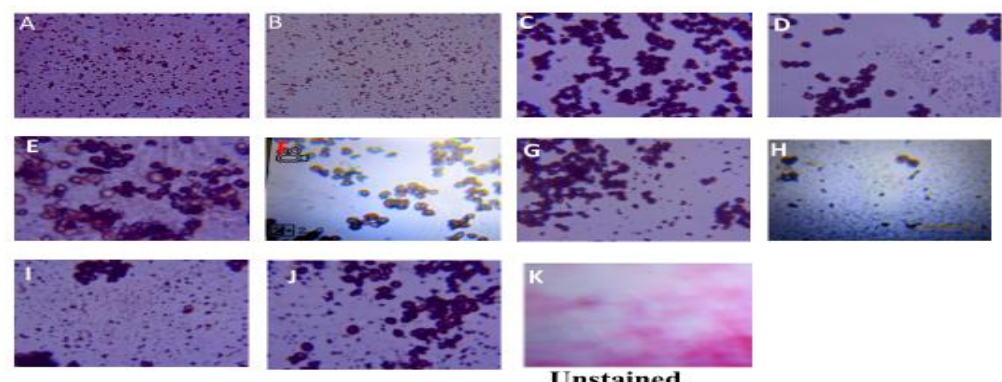

Figure 3. Micrographs of oil accumulation surrounding the cells of screened fungal isolates A-BW1, B-BW3, C-BW4, D-BW7, E-PW3, F-PW8, G-PW10, H - PW16, I - T, reesei, $\mathbf{J}-M$. circinelloides, $\mathbf{K}-\mathrm{PW} 4$.

Figure 3. Micrographs of oil accumulation surrounding the cells of screened fungal isolates A - BW1, B BW3, C - BW4, D - BW7, E - PW3, F-PW8, G - PW10, H - PW16, I - T. reesei, J - M. circinelloides, $\mathbf{K}$ - Unstained

Quantitative Determination of lipid content of the screened isolates

The amount of oil produced by the strains that were positive for oil accumulation was measured as a fraction of cell weight/volume of the medium. The lipid yield of the strains, BW 1, BW 3, BW 4, BW 7,
PW 3, PW 8, PW 10, PW 16, M. circinelloides and T. reesei were determined (Table $\mathbf{1}$ ). Out of these 10 strains, the highest oil producers were $M$. circinelloides $8.05 \mathrm{~g} / \mathrm{L} ;(41.3 \%)$, T. reesei $4.2 \mathrm{~g} / \mathrm{L}$ (28.7\%), BW4 $3.18 \mathrm{~g} / \mathrm{L} ;(35.3 \%)$, PW10 $5.27 \mathrm{~g} / \mathrm{L}$; (34.6\%) PW16 $2.26 \mathrm{~g} / \mathrm{L} ;(29.9 \%)$.

Table 1: Lipid Yield Estimation for screened isolates.

\begin{tabular}{llll}
\hline Isolate & Dry cell weight(g/L) & Lipid yield (w/v) & Lipid content (\%) \\
BW1 & 5.13 & 1.47 & 28.6 \\
BW3 & $7.43 \pm 0.00$ & $1.90 \pm 0.01$ & 25.6 \\
BW4 & 9.00 & 3.18 & 35.3 \\
BW7 & $7.87 \pm 0.001$ & $2.23 \pm 0.00$ & 28.3 \\
PW3 & 7.20 & 2.43 & 33.7 \\
PW8 & $2.27 \pm 0.00$ & $0.423 \pm 0.01$ & 18.6 \\
PW10 & 15.20 & 5.27 & 34.6 \\
PW16 & 7.56 & 2.264 & 29.9 \\
Mucor circinelloides & 19.65 & 8.05 & 41.3
\end{tabular}


Nsa et al./ Nig. J. Biotech. Vol. 37 Num. 1 : 138-149 (June 2020)

Table 2: Molecular identification of the screened isolates.

\begin{tabular}{llll}
\hline Isolate & Source & Isolate Identification & Accession Number \\
BW1/IYN75 & Brewery Wastewater & Candida tropicalis & MT3777705 \\
BW3/IYN76 & Brewery Wastewater & Aspergillus tubingensis & MT3777706 \\
BW4/IYN74 & Brewery Wastewater & Aspergillus sp. & MT3777704 \\
BW7/1YN71 & Brewery Wastewater & Trichosporon loubieri & MT3777701 \\
PW3/1YN77 & Ogi Wastewater & Candida tropicalis & MT3777707 \\
PW8/1YN72 & Ogi Wastewater & Aspergillus flavus & MT3777702 \\
PW10/IYN73 & Ogi Wastewater & Aspergillus fumigatus & MT3777703 \\
PW 16/IYN78 & Ogi Wastewater & Candida tropicalis & MT3777708 \\
IYN13 & Soil & Mucor circinelloides & MT421897 \\
IYN15 & Soil & Trichoderma reesei & MT421898 \\
\hline
\end{tabular}

\section{Molecular identification of the fungal isolates} With the BLASTn tool, the DNA sequences of the ITS$1,5.8 \mathrm{~S}$ rRNA and ITS2 regions for each isolate were compared to the ITS1 sequences deposited in NCBIGenBank and a species match was called by an identity threshold of $>99 \%$. The sequences of the fungal isolates obtained from this study have been deposited in GenBank and their Accession numbers issued have been indicated (Table 2). The BW1, BW3, BW4 and BW7, PW3, PW8, PW10 and PW16 were identified as Candida tropicalis, Aspergillus tubingensis, Aspergillus sp., Trichosporon louberi, Candida tropicalis, Aspergillus flavus, Aspergillus fumigatus and Candida tropicalis, respectively.

FAMES analysis of the oil extracts obtained from the isolates

Based on the lipid production yield, M. circinelloides, T. reesei, PW 10 (Aspergillus fumigatus) and BW 4 (Aspergillus sp.) were selected for FAMES analysis. FAMES profiles for the isolates $M$. circinelloides and $T$. reesei were determined with glucose and sucrose while only glucose was used for PW 10 and PW 4.

T. reesei and M. circinelloides did not contain any short-chain FAMEs, caproic acid (C6:0) and caprylic (C8:0) when either sucrose or glucose was the substrate. The principal fatty acid methyl ester of lipid extracts from $T$. reesei $(85.94 \%, 87.31 \%)$ and M. circinelloides $(50.41 \%, 87.33 \%)$ was the longchain fatty acid- palmitoleic acid (C16:1). Whereas, its yield in Aspergillus fumigatus and the other Aspergillus sp. (BW4) was $1.70 \%$ and $1.32 \%$ respectively. Palmitic acid (C16:0) was the second most abundant long-chain fatty acid from $T$. reesei (7.11\%) and M. circinelloides (6.37\%). The other long-chain FAMEs were present at concentrations of $3-4 \%$ in $T$. reesei and $3-11 \%$ in $M$. circinelloides (Table 3). $A$. fumigatus was enriched in the medium-chain, capric acid (C10:0) (95.37\%) and had a low concentration of long-chain FAMEs ( 3\%). The other Aspergillus sp. (BW4) had lauric acid as the principal FAME $(69.99 \%), 16.27 \%$ of mediumchain FAME, capric acid and $13.36 \%$ long-chain FAMEs. The Oleic acid and elaidic acid concentrations were highest in Aspergillus sp. (BW4). Among the four species, Aspergillus sp. (BW4) had the greatest number of FAMEs undetected (caproic acid, caprylic acid, linoleic acid, linoleaidic acid, arachidic acid, linolenic acid heptadecanoic acid) whereas $A$. fumigatus had 17 FAMEs. Tridecanoic acid was only present in $M$. circinelloides. The extracts from $T$. reesei and $M$. circinelloides were high in MUFA while 
Nsa et al./ Nig. J. Biotech. Vol. 37 Num. 1 : 138-149 (June 2020)

the Aspergillus species were high in saturated fatty

acids.

Table 3: FAMEs profile of acid-catalyzed transesterified oil extracts of the fungal strains

\begin{tabular}{|c|c|c|c|c|c|c|}
\hline \multirow[t]{2}{*}{ FAMEs (Mg/L) } & \multicolumn{2}{|c|}{ T. reesei } & \multicolumn{2}{|c|}{ M. circinelloides } & \multirow{2}{*}{$\begin{array}{l}\text { A. fumigatus } \\
\text { Glucose }\end{array}$} & \multirow{2}{*}{$\begin{array}{l}\text { Aspergillus sp. } \\
\text { Glucose }\end{array}$} \\
\hline & Sucrose & Glucose & Sucrose & Glucose & & \\
\hline Caproic Acid (C6:0) & - & - & - & & 0.71 & - \\
\hline Caprylic Acid (C8:0) & - & - & - & & 0.05 & - \\
\hline Capric Acid (C10:0) & 3.37 & 1.14 & 15.11 & 0.91 & 95.37 & 16.26 \\
\hline Undecanoic Acid (C11:0) & - & 0.16 & 1.80 & - & 0.04 & 0.38 \\
\hline Tridecanoic Acid (13:1) & ND & ND & - & 0.38 & ND & \\
\hline Lauric Acid (C12:0) & 2.75 & 1.05 & 10.43 & 1.08 & 0.18 & 69.99 \\
\hline Myristic Acid (C14:0) & 0.059 & 1.70 & 0.83 & 0.23 & 0.10 & 0.18 \\
\hline Myristoleic Acid (14:1) & 1.31 & 0.47 & 6.42 & 0.50 & 0.03 & - \\
\hline Pentadecanoic Acid (C15:0) & 0.39 & 0.08 & 1.44 & 0.07 & 0.14 & 0.21 \\
\hline Palmitic Acid (C16:0) & 0.38 & 7.11 & 2.47 & 6.37 & 0.13 & 0.72 \\
\hline Palmitoleic Acid (C16:1) & 85.94 & 87.31 & 50.41 & 87.33 & 1.70 & 1.32 \\
\hline Heptadecanoic Acid (C17:1) & 1.76 & 1.25 & 5.98 & 1.58 & 0.03 & - \\
\hline Stearic Acid(C18:1) & 0.51 & 0.58 & - & 0.67 & 0.01 & 0.80 \\
\hline Linoleic Acid (C18:2) & ND & - & 1.55 & 0.17 & & \\
\hline Elaidic Acid (C18:1) & 2.43 & 0.21 & 0.64 & 0.21 & 0.07 & 6.81 \\
\hline Linolelaidic Acid (C18:2) & 0.23 & 0.35 & 1.74 & 0.39 & 0.33 & - \\
\hline Arachidic Acid (20:0) & 0.30 & - & 1.19 & - & 0.01 & - \\
\hline Linolenic Acid (C18:3) & 0.41 & - & - & - & 0.10 & - \\
\hline Oleic Acid(C18:1) & 0.13 & 0.08 & - & 0.11 & 0.01 & 0.34 \\
\hline Saturated \% & 39.2 & 11.57 & 9.53 & 11.30 & 96.45 & 88.81 \\
\hline Monounstaurated \% & 59.2 & 88.43 & 90.06 & 88.53 & 1.98 & 9.20 \\
\hline Polyunsaturated \% & 1.5 & 0.0 & 0.41 & 0.16 & 0.33 & 2.00 \\
\hline
\end{tabular}


Nsa et al./ Nig. J. Biotech. Vol. 37 Num. 1 : 138-149 (June 2020)

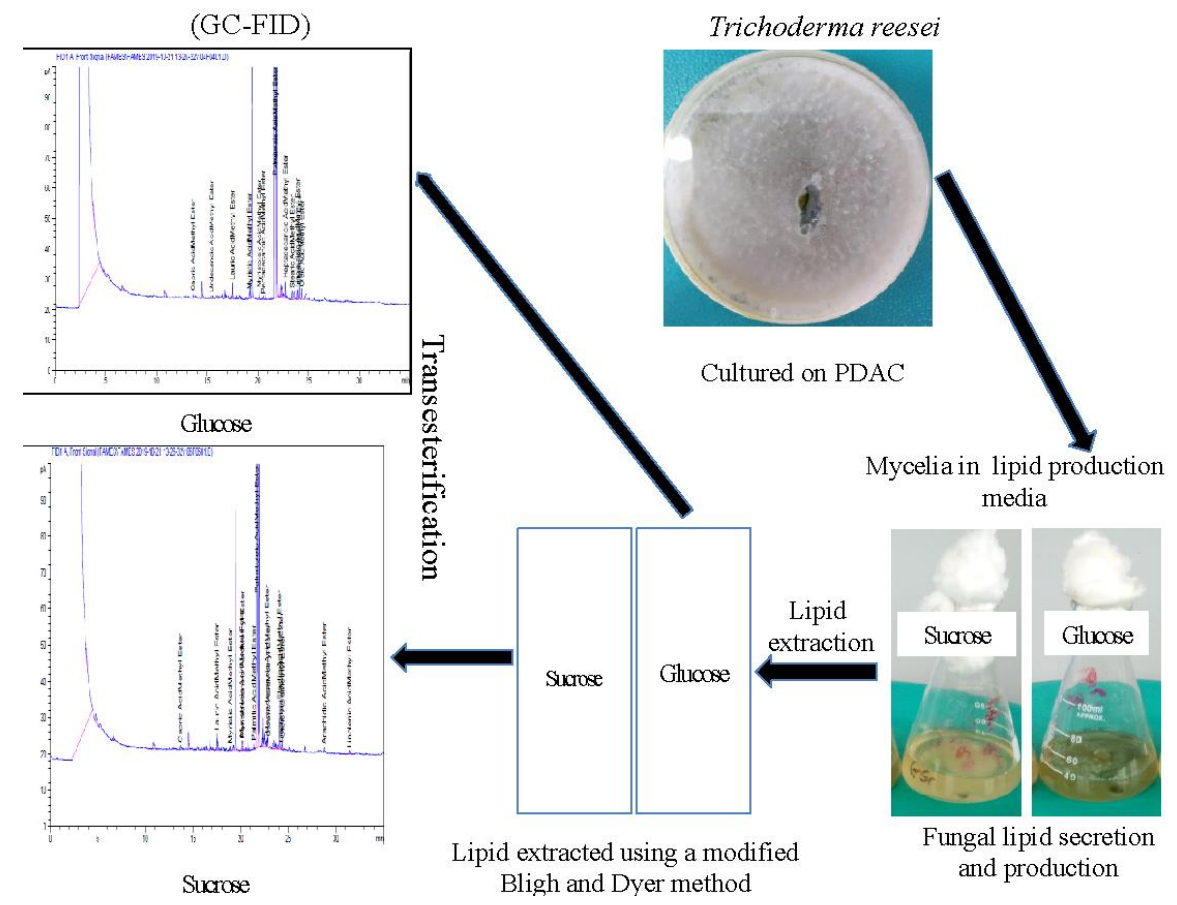

Figure 4. Flowchart of oil production for Trichoderma reesei from its cultivation on potato dextrose agar to oil extraction and GC-FID chromatogram of the FAMEs.

\section{Discussion}

The prospects of isolating microorganisms from wastewater and soil for the production of microbial oils have been extensively studied (Muniraj et al., 2015; Muniraj et al., 2017; Sheerin et al., 2017). A total of 25 fungal strains were isolated from fermented maize (ogi) wastewater (16) and sorghum-based brewery wastewater (9). The new fungal isolates and two existing laboratory fungal strains, M. circinelloides and T. reesei isolated from the soil were screened for oleaginicity based on the accumulation of lipid in their globules and also the quantification of oil produced by Modified Sudan Black B Method (Muniraj et al., 2017; Thanchaeron et al. 2017) (Table 1) Eight selected strains that had at least $20 \%$ oil accumulation capability were identified by culture-based methods and ITS-rDNA sequencing to be Aspergillus fumigatus (PW8), Aspergillus

flavus (PW10), Candida tropicalis (PW16) and Aspergillus tubingensis (PW3), Trichosporon luoberi (BW7), Aspergillus sp. (BW4) and Candida tropicalis (BW1 and BW3). It was not surprising that Aspergillus spp. and C. tropicalis were the most commonly encountered oleaginous microbes from the fermented beverage wastewater as they have been reported as part of the fungal community involved in the fermentation of maize (ogl) (Anumudu et al., 2018).
C. tropicalis isolated from a cassava processing wastewater source had a similar lipid yield of $29.4 \%$ (Ashika et al., 2017) to the C. tropicalis isolates from our study (BW1-28.6\%, PW3-33.7\%, PW 16 $29.9 \%$ ). The other oleaginous yeast identified from brewery wastewater was Trichosporon loubieri, other species T. cutaneum, T. fermantans, $T$. capitatum and $T$. oleaginosus are known to accumulate lipids (Zhu et al., 2008; Wu et al., 2011; Chen et al., 2013; Kourist et al., 2015). Oleaginous isolates of Aspergillus genera including $A$. flavus, $A$. fumigatus and $A$. tubingensis have been reported from environmental samples (Abu-Elreesh and AbdEl-Haleem, 2014; Kadhim and Alrubayae, 2019 Shafiq, 2017; Khahim and Alrubayae, 2019; AbuElreesh and Abd- El-Halleem, 2014). This study reports the isolation of $A$. tubigensis from brewery wastewater accumulating $25.6 \%$ of its biomass as lipids. Intasit et al., 2019 reported a strain of $A$. tubigensis (TSIP9) accumulating $25.57 \%$ lipid when cultivated on a non-sterile palm empty fruit branch biomass. It was not surprising that $A$. flavus had the lowest amount of lipids as it had the least dry cell weight. It was observed that the soil isolate, $M$. circinelloides had the highest lipid content (41.3\%), and biomass $(19.65 \mathrm{~g} / \mathrm{L})$. T. reesei had a yield of $28.7 \%$ with a dry cell weight (DCW) of $14.60 \mathrm{~g} / \mathrm{L}$ comparable to a report where $T$. reesei isolated from wood could produce $30 \mathrm{~g} / \mathrm{L}$ and $32.4 \%$ of DCW 
Nsa et al./ Nig. J. Biotech. Vol. 37 Num. 1 : 138-149 (June 2020)

(Bharathiraja et al., 2017). The potential of strains of $M$. circinelloides and $T$. reesei as oleaginous fungi and feedstock for biodiesel production has been widely reported (Bhanja et al., 2014; Qaio et al., 2018; Zhao et al., 2018; Kumar et al., 2014; Bharathiraja et al., 2017).

FAMEs profiles were determined for the four fungal isolates M. circinelloides, (glucose, sucrose) T. reesei (glucose, sucrose) Aspergillus fumigatus PW10 (glucose) Aspergillus sp. (BW 4) (glucose). Transesterified oil extract from $M$. circinelloides and $T$. reesei in this study showed higher amounts of unsaturated fatty acid while the two Aspergillus species examined were high in saturated fatty acids. Palmitoleic acid was the most abundant fatty acid methyl ester of $M$. circinelloides and $T$. reesei regardless of the cultivation medium being glucose or sucrose. However, the use of glucose as substrate enhanced the recovery of palmitic acid methyl ester from $0.38 \%$ to $7.11 \%$ and $2.47 \%$ to $6.37 \%$ in $M$. circinelloides and $T$. reesei respectively. Overall, for $T$. reesei, the FAMES profile generated from sucrose and glucose were not overly different from each other, but in M. circinelloides, lauric acid and capric acid were enriched when sucrose was the substrate.

FAMEs profile was dependent on the type of carbon source - glucose or sucrose (Table 3; Fig. 4). The effect of carbon sources on fatty acid composition and lipid accumulation in oil-producing moulds have been reported (Liu et al., 2010; Thanaa and Dina, 2014). The major fatty acid produced in the $M$. circinelloides is palmitoleic acid which accounted for $50.41 \%$ and $87.3 \%$ in sucrose and glucose respectively. In $T$. reesei, palmitoleic acid methyl ester was the major fatty acid , having $85.94 \%$ and $87.31 \%$ with sucrose and glucose respectively. The palmitoleic acid composition of $M$. circinelloides and $T$. ressei has been reported to be indicative of its use as an excellent feedstock for the production of single-cell oil (Bhanja et al., 2014).

The finding here that medium-chain saturated capric acid was the dominant FAME in $A$. fumigatus and the second most abundant in Aspergillus sp. (BW4) contrasts the report from Asci et al. (2020) who showed that the FAMEs profile of the six $A$. fumigatus strains studied were dominated by long-chain fatty acids and suitable for use as biodiesel.

In this study, Palmitoleic acid was produced at much higher concentrations by $M$. circinelloides and $T$. reesei at concentrations higher than observed from plant oils like Macadamia (28\%) (Matthan et al.,
2009). Palmitoleic acid, the most abundant FAME in $T$. reesei and $M$. circinelloides is known to improve biodiesel properties (Knothe, 2009).

M. circinelloides was the most promising fungus for use as biodiesel but its profile ratios need to be improved for such use. In general, hexadecanoic (palmitic, C16.0), octadecanoic acid (stearic, C18:0) octadecenoic (oleic, C18:1) octadecatrienoic acid (linolenic, C18:3) and octadecenoic (linoleic, C18:2) fatty acids are present among other FAMEs in different quantities in biodiesel (Li et al., 2019). In comparison with the profiles from M. circinelloides strains used as feedstock for biodiesel (Qiao et al., 2018; Reis et al., 2011; Carvalho et al., 2017) one or more of these FAMEs were absent or present at very low levels in the oleaginous fungi for such consideration. Its relevant long-chain FAMEs profile with sucrose, was palmitic $(2.47 \%)$, linoleic $(1.55 \%)$, differed from that of the FAMEs profile of palmitic $(6.37 \%)$, stearic $(0.67 \%)$, oleic $(0.11 \%)$, linoleic $(0.17 \%)$, and linolenic $(0.17 \%)$ from glucose.

The FAMEs profiles of the oleaginous strains investigated had low levels or total absence of these necessary FAMEs suggesting that the oils extracted from these isolates under the culture conditions are not fit for use as biodiesel. Further optimization of cultivation and lipid extraction conditions and genetic engineering may improve fatty acid methyl ester profiles of the most promising isolate $M$. circinelloides for its use as feedstock for biodiesel production.

The FAMEs profile of the fungal isolates from this work shows that they would be a good feedstock for the production of pharmaceuticals and cosmetics. Lauric acid and capric acid are used in the cosmetic industry as emollients, dispersing agents for other chemicals, solvents and antioxidants. They can inhibit the growth of Propionibacterium acne, act as intestinal anti-inflammatory and alleviate oxidative stress (Huang et al., 2014; Lee and Kang, 2017). Palmitoleic acid is used to improve cold flow and also acts as a stabilizer for biodiesel (Knothe, 2010). These fatty acids have also been reported to have effects on the inhibition of Candida albicans (Clément et al., 2007; Murzyn et al., 2010).

\section{Conclusion}

In conclusion, among all the oleaginous species assayed, the FAMEs profile of $M$. circinelloides contained a better composition of biodiesel relevant fatty acids although at low proportions. Optimization 
Nsa et al./ Nig. J. Biotech. Vol. 37 Num. 1 : 138-149 (June 2020)

of culture conditions, transesterification, extraction procedures and genetic manipulation for this isolate might be recommended for future research for obtaining better biodiesel production.

\section{Acknowledgements}

We thank Mr. Anichie Phil-Ebosie for sharing with us his ideas on biodiesel and Deborah Ogunleye for her talk on microbial oil given in the departmental seminar of the Department of Microbiology, University of Lagos.

\section{References}

Altschul, S.F., Gish, W., Miller, W., Myers, E.W. and Lipman, D.J. (1990). Basic Local Alignment Search Tool. J. Mol. Biol. 215:403-410.

Anumudu, C., Omeje, K., Iyeoma, F. and Obinwa, G. (2018). Microbial succession pattern in Ogi fermentation. Int. J. Adv. Res. Biol. Sci. 5(7): 247251. DOI:

http://dx.doi.org/10.22192/ijarbs.2018.05.07.019.

Asci, F., Aydin, B., Akkus, U., Unai, A., Erdogmus, F., Korcan, E. Jahan, I. (2020). Fatty acid methyl esters of Aspergillus fumigatus isolated from fruit pulp for biodiesel production. Bioengineered 11: 408-415.

Ashika, S., Kiruthika, T., Ashokkumar, K., Suraj, M. and Uthandi, S. (2017). Oleaginous Yeast from Sago Wastewater: Screening and Characterization of Candida tropicalis for Biolipid production. Madras Agric. J. 104 (7-9): 288-291.

Atabani, A.E., Silitong, A. S., Ong, H. C., Mahlia, T., Masjuki, H. H., Irfan, A. and Fayaz, H. (2013). Nonedible vegetable oils: A critical evaluation of oil extraction, fatty acid compositions, biodiesel production, characteristics, engine performance and emissions production. Renew. Sust. Energ. Rev. 18: 211-245.

Bhanja, A., Minde, G., Magdum, S. and Kalyyanraman, V. (2014). Comparative studies of Oleaginous Fungal Strains (Mucor circinelloides and Trichoderma ressel) for effective wastewater treatment and Bio-oil production. Biotechnol. Res. Int. 2014;2014:479370. doi:10.1155/2014/479370.

Bharanthiraja, B., Chakravarthy, M., Kumar Ranjith, R., Yuvaraj, D., Jayamuthunagi, J., Kumar, P. and Palani, S. (2014). Biodiesel production using chemical and biological methods- A review. of process, catalyst, acyl acceptor, source and process variables. Renew. Sust. Energ. Rev. 38: 368-382.

Bharathiraja, B., Sowmya, V., Sridharan S., Yuvaraj., Jayamuthunagi, J. and Praveenkumar, R. (2017). Biodiesel production from microbial oil derived from wood isolate Trichoderma reesei. Bioresour. Technol. 239: 538-541.

Calvey, C. H., Su, Y. K., Willis, L. B., McGee, M. and Jeffries, W. (2016). Nitrogen limitation, oxygen limitation and lipid accumulation in Lipomyces starkeyi. Bioresour. Technol. 200: 780-778.

Carvalho, A., Conceição, L., Silva, J., Perez, V. and Castro, H. (2017). Biodiesel production from Mucor circinelloides using ethanol and heteropolyacid in one and two-step transesterification. Fuel 202: 503511. 10.1016/j.fuel.2017.04.063.

Chen, X., Huang, C., Yang, Y., Xiong, L., Chen, X. and $\mathrm{Ma}$, L. (2013). Evaluating the effect of medium composition and fermentation condition on the microbial oil production by Trichosporon cutaneum on corncob acid hydrolysate. Bioresour. Technol. 143: 18-24.

Clément, M., Tremblay, J., Lange, M., Thibodeau, J. and Belhumeur, P. (2007). Whey-derived free fatty acids suppress the germination of Candida albicans in vitro, FEMS Yeast Res. 7(2):276285, https://doi.org/10.1111/j.1567-

1364.2006.00166.x

Gavrilescu, M. and Chisti, Y. (2005). Biotechnologya sustainable alternative for the chemical industry. Biotechnol. Adv. 23 (7-8):471-99.

Gienteka, L., Peng, F., Xu, F. and Sun, R. (2017). Lipid production and analysis in Candida spp., isolated from marine waters. FEBS 23: 56-62.

Hall, T.A. (1999). BioEdit: a user - friendly biological sequence alignment editor and analysis program for Windows 95/98/NT. Nucl. Acids Symp. Ser. 41: 9598.

Hanna, M. A. (1991). Biodiesel production: A review. Bioresour. Technol. 70: 1-15.

Huang, W., Tsai, T., Chuang, L., Li, Y., Zouboulis, C. and Tsai, P. (2014). Antibacterial and antiinflammatory properties of capric acid against Propionibacterium acnes: A comparative study with Lauric acid. J. Dermatol. Sci. 73: 232-240. 
Nsa et al./ Nig. J. Biotech. Vol. 37 Num. 1 : 138-149 (June 2020)

Intasit, R., Yeesang, J. and Cheirsilp, B. (2019). Biological pretreatment of Empty Fruit Bunch (EFB) Using Oleaginous Aspergillus tubigensis (TSIP9. J. Water Environ. Tech. 17(4). 244-250.

Kalayasiri, P., Jayashoke, N. and Krisnangkura, K. (1996). Survey of seed oils for use as diesel fuels. J. Am. Oil Chemi.' Soc. 73(4): 471-474.

Karatay, M. and Domez, L. (2010). Production of lipid from Oleaginous species of Candida. J. Microbiol. Biotechn. 220: 38-36.

Kadhim, F. and Alrubayae, I. (2019). Study of Lipase production and Lipid accumulation of Oleaginous Fungi isolated from oil-rich soil in Basrah. SJMR 3 (12): 123-127.

Khot, M., Kamat, S., Zinjarde, S., Pant, A., Chopade, B. and Ravikumar, A. (2012). Single-cell oil of oleaginous fungi from the tropical mangrove wetlands as a potential feedstock for biodiesel. Microb. Cell Fact. 11:71.

Knothe, G. (2010). Biodiesel Derived from a Model Oil Enriched in Palmitoleic Acid, Macadamia Nut Oil. Energy Fuels 24: 2098-2103.

Kolouchova, I., Sigler, K., Schreiberova, O., Masak, J. and Rezanka, T. (2015). New yeastbasedapproaches in the production of palmitoleic acid. Bioresour. Technol. 152: 726-734.

Kosa, M. and Ragauskas, A. (2011). Lipids from heterotrophic microbes: advances in metabolism research. Trends Biotechnol. 29(2): 53-61.

Kourist R, Bracharz F, Lorenzen J, et al. (2015). Genomics and Transcriptomics Analyses of the OilAccumulating Basidiomycete Yeast Trichosporon oleaginosus: Insights into Substrate Utilization and Alternative Evolutionary Trajectories of Fungal Mating Systems. mBio. 2015;6(4): e00918.

Kumar, R., Kumaran, B., Balashanmugam. (2014). Production of Cellulase Enzyme by Trichoderma ressei Cef 19 and its application in the production of Bioethanol. PJBS 17(5): 735-739.

Lee, S. and Kang, K. (2017). Function of capric acid in cyclophosphamide-induced intestinal inflammation, oxidative stress and barrier function in pigs. Sci. Rep. 7: 16530.

Li, Q., Du, W., Liu, D. (2008). Perspectives of microbial oils for biodiesel production. Appl.
Microbiol. Biotechnol. doi:10.1007/s00253-008-1625-9.

80(5):749-756.

Li, F., Liua, Z., Nia, Z. and Wang, H. (2019). Effect of Biodiesel Components on its Lubrication Performance. J. Mater. Res. Technol. 8(5): 36813687. https://doi.org/10.1016/j.jmrt.2019.06.011

Liu, G., Lin, Q., Jin, X., Wang, X. and Zhao, Y. (2010). Screening and fermentation optimization of microbial lipid-producing moulds from forest soils. Afr. J. Microbiol. Res. 4(14): 1462-1468.

Matthan, N. R., Dillard, A., Lecker, J. L., Ip, B., \& Lichtenstein, A. H. (2009). Effects of dietary palmitoleic acid on plasma lipoprotein profile and aortic cholesterol accumulation are similar to those of other unsaturated fatty acids in the F1B golden Syrian hamster. J. Nutr. 139(2), 215-221. https://doi.org/10.3945/jn.108.099804.

Meher, L. C., Vidya Sagar, D. and Naik, S. N. (2006). "Technical aspects of biodiesel production by transesterification-a review. Renew. Sust. Energ. Rev. 10(3): 248-268.

Meng, X., Yang, M., Xu, X., Zhang, L., Ni, J. and Xian, M. (2009). Biodiesel production from oleaginous microorganisms. Renew. Energy 34:1-5.

Muniraj, I., Liwen, X., Zhenhu, H. and Zhan, X. (2017). Screening and Characterization of Oleaginous Fungi from Irish Soil for Growth under Low carbon substrates. Int. J. Curr. Microbiol. Appl. Sci. 6(12): 772-781.

Muniraj, I., Xiao, L., Liu, H. and Zhan, X. (2015). Utilisation of potato processing wastewater for microbial lipids and a-linolenic acid production by oleaginous fungi. J. Sci. Food Agric. 95: 3084-3090.

Murzyn, A. and Krasowska, A., Stefanowicz, P., Dzaidkowiec, Dorota, D. and Lukaszewics, M. (2010). Capric acid secreted by $S$. boulardii inhibits C. albicans filamentous growth, Adhesion and Biofilm Formation. PLoS One 5(8): e12050.

Nsa, I.Y., Kareem, R.O. Aina, O.T., and Akinyemi, B.T.SEEp (2020). Isolation of an Emerging Thermotolerant Medically Important Fungus, Lichtheimia ramosa from soil. Afr. J. Microbiol. Res. Vol. X (X) DOI:10.5897/AJMR.

Peberdy, J. (1994). Protein secretion in filamentous fungi- trying to understand a highly productive black box. Trends Biotechnol. 12: 50-57. 
Nsa et al./ Nig. J. Biotech. Vol. 37 Num. 1 : 138-149 (June 2020)

Punt, P. J., van Biezen, N., Conesa, A., Albers, A., Magnus, J. and den Hondel, C. (2002) Filamentous fungi as cell factories for heterologous protein production. Trends Biotechnol. 20: 200-206.

Qaio, W., Tao, J., Luo, Y., Tang, T., Miao, J. and Yang, Q. (2018). Microbial oil production from solidstate fermentation by a newly isolated oleaginous fungus, Mucor circinelloides Q531 from mulberry branches. R. Soc. Open Sci. 5: 180551.

Ratledge, C. (2002). Regulation of lipid accumulation in oleaginous microorganisms. Biochem. Soc. Trans. 3(6): 1047-1050.

Ratledge, C. and Wynn, J. (2002). The Biochemistry and Molecular Biology of Lipid Accumulation in Oleaginous Microorganisms. Adv. Appl. Microbiol. 51:1-52.

Reis, C. E. R., Hu, B., \& Zhang, J. (2011). Direct biodiesel production from mucor circinelloides fungi. In 11AIChE - 2011 AIChE Annual Meeting, Conference Proceedings (11AIChE - 2011 AIChE Annual Meeting, Conference Proceedings).

Shafiq A. (2017). Biodiesel production by oleaginous fungi before and after exposing of U.V light. Int. J. ChemTech Res. 10(12). 357-363.

Sawangkeaw, R. and Ngamprasertsith, S. (2013). A review of lipid-based biomasses as feedstocks for biofuels production. Renew. Sust. Energ. Rev. 24:97-108.

Sheerin, T., Turian, C. and Trumen, B. (2017). Screening of moulds from slaughterhouse wastewater samples for oleaginous testing. J. Appl. Microbiol. 3: 15-18.

Thakur, S. Prapulla, G. and Karanth, G. (1987). Microscopic Observation of Sudan Black B staining to Monitor Lipid Production by Microbes. J. Chem. Technol. Biotechnol. 42: 129-134.

Thanaa, A and Dina, H. (2014). Optimization of culture conditions for the highest Lipid production from some Oleaginous Fungi for Biodiesel Preparation. Asian J. Appl. Sci. 2(5): 600-609.

Thancharoen, K., Malasari, Auttaporn, M., Leamsingkorn, W. and Boonyalit, P. (2017). Selection of Oleaginous yeasts with lipid accumulation. IJPMBS 6(2): 53-57.
Thevenieau, F. and Nicaud, J. (2013). "Microorganisms as sources of oils". OL CORPS GRAS LI 20(6):603.

Ventaka, S. and Ventaka, M. (2011). Biodiesel production from isolated oleaginous fungi Aspergillus sp. using corncob waste liquor as a substrate. Bioresour. Technol. 102(19): 9286-9290.

Vicente, G., Baustista, F., Rodriguez, R., Gutierrez, J., Sadaba, I., Ruiz-Vazquez, M., Torres-Martinez, S. and Garre, V. (2009). Biochem. Eng. J. 48: 22-27.

Weimann, E., MAysa Braga, B., Murata, G., Bortolon, J., Dermagos, A. and Curl, R. and Hatanka, B. (2018). Topical anti-inflammatory activity of palmitoleic acid improves wound healing. PLoS One 13(10): e0205338.

Wösten H. (2019). Filamentous fungi for the production of enzymes, chemicals and materials. Curr. Opin Biotech. 59: 65-70. DOI: 10.1016/j.copbio.2019.02.010.

Wu, H., Li, Y., Chen, L. and Zong, M. (2011). Production of microbial oil with high oleic acid content by Trichosporon capitatum. Appl. Energy 88: 138-142.

Yap, T. Y., Abdullah, N. F., and Basri, M. (2011). Biodiesel production via transesterification of palm oil using $\mathrm{NaOH} / \mathrm{Al}_{2} \mathrm{O}_{3}$ Catalysts. Sains Malays. 40 (6): 587-594.

Zhang, X., Yan, S., Tyagi, R.D., Drogui, P. and Surampalli, R.Y. (2014). Ultrasonication assisted lipid extraction from oleaginous microorganisms. Bioresour. Technol. 158: 253-261.

Zhao, H., Liu, X., Zhan, T. and He, J. (2018). Production of cellulase by Trichoderma reesei from pretreated straw and furfural residues. RSC Adv. 8: 36233-36238.

Zhu, L.Y., Zong, M.H. and Wu, H. (2008) Efficient lipid production with Trichosporon fermentans and its use for biodiesel preparation. Bioresour. Technol. 99: 7881-7885. 